Binghamton University

The Open Repository @ Binghamton (The ORB)

$3-31-2020$

Comparison of colorimetric analyses to determine cortisol in human sweat

Ethan Tu

Peter Pearlmutter

Michelle Tiangco

Gia Derose

Lina Begdache

See next page for additional authors

Follow this and additional works at: https://orb.binghamton.edu/hws_fac

Part of the Medicine and Health Sciences Commons 
Authors

Ethan Tu, Peter Pearlmutter, Michelle Tiangco, Gia Derose, Lina Begdache, and Ahyeon Koh 


\title{
Comparison of Colorimetric Analyses to Determine Cortisol in Human Sweat
}

\author{
Ethan Tu, Paul Pearlmutter, Michelle Tiangco, Gia Derose, Lina Begdache, and Ahyeon Koh*
}

Cite This: ACS Omega 2020, 5, 8211-8218

Read Online

ABSTRACT: Colorimetric analysis, which relies on a chemical reaction to facilitate a change in visible color, is a great strategy for detecting cortisol, which is necessary to diagnose and manage the wide variety of diseases related to the hormone, because it is simple in design, inexpensive, and reliable as a standard cortisol analysis technique. In this study, four different colorimetric cortisol analyses that use various chromogens, which include sulfuric acid, Porter-Silber reagent, Prussian blue, and blue tetrazolium, are studied. Modifications to the classic Porter-Silber method are made by increasing the carbon content of the alcohol and adding gold nanoparticles, which result in a twofold increase in reaction rate and a slight decrease in the limit of detection (LoD). After a comparison of the reaction rate, $\mathrm{LoD}$, dynamic range, characteristic peaks, and color stability of all methods, blue tetrazolium demonstrates a low LoD $(97 \mathrm{ng} / \mathrm{mL})$, broad dynamic range $(0.05-2$

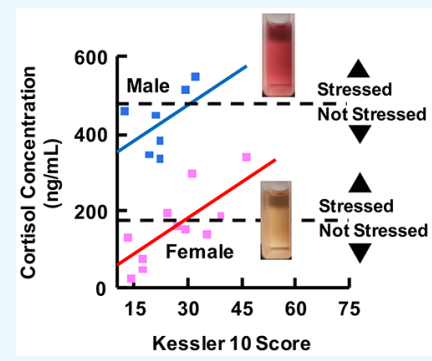
$\mu \mathrm{g} / \mathrm{mL}$ ), and quick reaction rate (color development as fast as $10 \mathrm{~min}$ ), which are well within the requirements for human biofluids. Cortisol in artificial saliva and sweat and in human sweat was determined while confirming that no excipients or other biomarkers interfered with the reactions. Twenty-one human sweat samples were tested using blue tetrazolium and revealed a significant difference between male and female apocrine cortisol concentrations and showed a highly significant difference between apocrine and eccrine cortisol concentrations. Colorimetric methods of cortisol can compete with existing electrochemical sensors because of their similar accuracy and detection range in certain wearable biosensor applications. The simplicity of colorimetric methods advances potential applications in skin-interfaced bio-electronics and point-of-care devices.

\section{INTRODUCTION}

Cortisol is an important biomarker that is secreted by the adrenal cortex to regulate blood pressure, glucose levels, and metabolism. ${ }^{1}$ Although cortisol is always present in human systems, its secretion outside of circadian dependencies is induced by psychological and emotional stress, which is why monitoring cortisol concentrations in the body is crucial in understanding stress-related diseases. ${ }^{2,3}$ Normal levels of cortisol range from 8 to $142 \mathrm{ng} / \mathrm{mL}$ in sweat, 40 to $250 \mathrm{ng} /$ $\mathrm{mL}$ in plasma, and 1 to $11 \mathrm{ng} / \mathrm{mL}$ in saliva, with the highest concentrations occurring during the morning and slowly decreasing throughout the day. ${ }^{4-6}$ Using any biofluid to identify and track abnormal cortisol concentration can help patients and caregivers manage a variety of diseases such as Cushing's syndrome and Addison's disease, which cause characteristically high or low levels of cortisol, respectively. ${ }^{7,8}$

The current gold standard in cortisol analysis uses an enzyme-linked immunosorbent assay (ELISA). However, colorimetric reactions also have been studied historically to determine cortisol concentrations. Early studies report fluorescent reactions among a number of steroids with concentrated sulfuric acid. ${ }^{9-11}$ Each steroid-acid mixture has similar but unique absorption spectra and obeys Beer's law. Zimmermann's reagent was adopted later on as a spot test for steroids and replaced pure sulfuric acid. ${ }^{12}$ In addition to greater accuracy and specificity, the absorption spectra were more easily differentiated. ${ }^{13}$ Corticosteroid specific tests first appeared with the Porter-Silber reagent in 1950, where a pale yellow hue was reported when hydrocortisone and cortisone were added to a solution that contains dilute sulfuric acid, methanol, and phenylhydrazine. ${ }^{14}$ The Porter-Silber reagent has been improved upon throughout the years, which results in faster reaction rates and a more robust color. ${ }^{15-17}$ Concurrent with the Porter-Silber reagent, Clark developed another corticosteroid specific reagent, which consists of dilute sulfuric acid and diphenylamine. ${ }^{18}$ Later on, reagents that used blue tetrazolium provided an even greater steroid assay, which lowered the reaction time to $10 \mathrm{~min}$ compared to the $90 \mathrm{~min}$ of Porter-Silber. ${ }^{19,20}$ Singh and Verma recently reported another method using cortisol's reduction properties to form Prussian blue from iron(III) and hexacyanoferrate(III), which is comparable to past formulations in terms of sensitivity, precision, and speed. ${ }^{21}$ With the rise of competitive protein binding immunoassay techniques, colorimetric reactions have been largely made obsolete, with little research published since the 1970s. However, with increased interest in cortisol

Received: February 4, 2020

Accepted: March 19, 2020

Published: March 31, 2020 


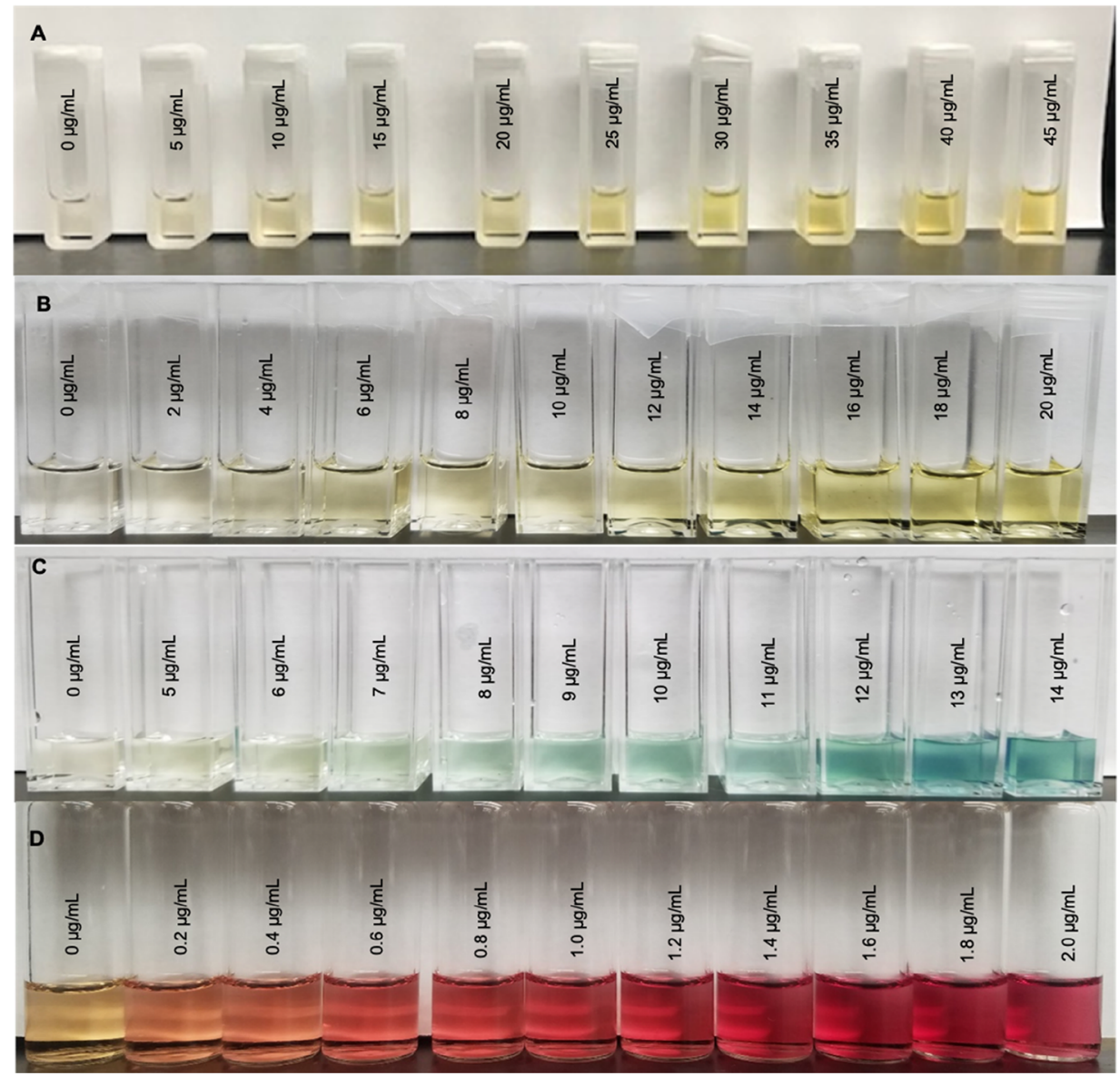

Figure 1. Optical images of color change with increasing concentration of cortisol: (A) sulfuric acid, (B) AuNP Silber-Porter, (C) Prussian blue, and (D) blue tetrazolium.

monitoring and detection in sweat and saliva with disposable biosensors, colorimetric analysis has become a prime candidate for such tasks that use simple and easy analytical analysis.

The in situ analysis of biofluids, such as saliva, urine, and sweat, has drawn significant attention over the years because of its noninvasive collection methods. In skin-interfaced and wearable devices, electrochemical techniques are preferred over competitive protein binding techniques because of their portability and potential point-of-care applications. ${ }^{22}$ Additionally, ELISA techniques and other immunoassay-based tests are expensive, are chemically complex, require a lengthy amount of time to complete, are difficult to scale up, and require cold-chain managements. ${ }^{23-26}$ Multiple research groups have worked to improve analyte stability, ${ }^{27}$ flexibility of electronic components, ${ }^{28,29}$ wearability, ${ }^{30-32}$ and sensitivity of electrochemical sensors. ${ }^{27,33,34}$ However, short shelf life, battery dependence, inflexible batteries, and relatively high cost for complete electronic systems currently prevent mainstream success. ${ }^{22}$ Colorimetric-based patch-type microfluidic devices have seen success with other biomarkers such as glucose, $\mathrm{pH}$, chloride, lactate, creatine, ammonia, and ethanol. ${ }^{31,35,36}$ Such techniques could provide a solution to the drawbacks of electrochemical systems, while retaining many benefits. The lack of electronic components can lower manufacturing cost and is capable of becoming disposable and offers more straightforward skin integration. ${ }^{32}$ Microfluidic channel design can also be tailored to accommodate continuous time analysis or single time analysis. ${ }^{37}$
Herein, we revisit and study the various colorimetric analyses of cortisol and improve its analytical performances that enable its use for wearable devices. Reactions reported by Zimmerman and Clark, as previously mentioned, were not studied because of the highly toxic components, which are not suitable for biosensor applications. Traditionally, both the Porter-Silber and Prussian blue methods have been slow to develop color, with reaction times that reach as long as $4 \mathrm{~h}$ at room temperature. The average reaction rate of the PorterSilber was accelerated by substituting ethanol for isopropanol and adding gold nanoparticles (AuNPs) as a catalyst. In addition, the reaction rate was enhanced by completing the Prussian blue reaction with cortisol in ethanol as opposed to deionized (DI) water. Overall, the blue tetrazolium method was found to be the most robust, which excels in both speed and accuracy when compared to other methods. The small-n human pilot study confirms that the cortisol in biofluid can be determined.

\section{RESULTS AND DISCUSSION}

In general, cortisol is believed to be a critical biomarker for monitoring stress levels. Therefore, a simple and sensitive colorimetric analysis of cortisol holds immense potential for daily-monitoring wearable devices. Four colorimetric analyses of cortisol detections that include sulfuric acid, Porter-Silber reagent, Prussian blue, and blue tetrazolium were investigated and further developed in this study. The mechanism of each colorimetric reaction was experimentally validated with optical 
Table 1. Comparison of Different Methods of Cortisol Detection

\begin{tabular}{|c|c|c|c|c|c|c|c|}
\hline method of detection & $\begin{array}{l}\text { characteristic } \\
\text { absorption peak } \\
(\mathrm{nm})\end{array}$ & $\begin{array}{c}\text { analytical } \\
\text { LoD } \\
(\mu \mathrm{g} / \mathrm{mL})\end{array}$ & $\begin{array}{l}\text { experimental } \\
\text { LoD }(\mu \mathrm{g} / \mathrm{mL})\end{array}$ & $\begin{array}{l}\text { dynamic range } \\
(\mu \mathrm{g} / \mathrm{mL})\end{array}$ & $\begin{array}{l}\text { time to color } \\
\text { development } \\
\quad(\mathrm{min})\end{array}$ & $\begin{array}{l}\text { visual color } \\
\text { change }\end{array}$ & color stability \\
\hline pure concentrated sulfuric acid & 290,394 , and 480 & 3.00 & 1.03 & $0-100$ & immediate & $\begin{array}{l}\text { clear to } \\
\text { florescent } \\
\text { yellow }\end{array}$ & unstable \\
\hline $\begin{array}{l}\text { Porter-Silber reagent with } \\
\text { AuNPs }\end{array}$ & 410 & 0.145 & 0.401 & $0-70$ & 60 & $\begin{array}{l}\text { clear to viscous } \\
\text { yellow }\end{array}$ & $\begin{array}{l}\text { stable up to } \\
24 \mathrm{~h}\end{array}$ \\
\hline $\begin{array}{l}\text { potassium } \\
\text { hexacyanoferrate(III) and } \\
\text { iron(III) reagent }\end{array}$ & 300 and 669 & 0.731 & 0.320 & undetermined & 45 & $\begin{array}{l}\text { pale yellow to } \\
\text { blue-green }\end{array}$ & $\begin{array}{l}\text { stable for at } \\
\text { least } 1 \text { week }\end{array}$ \\
\hline blue tetrazolium reagent & 510 & 0.097 & 0.214 & $0-1.5$ & $5-10$ & $\begin{array}{l}\text { pale yellow to } \\
\text { magenta }\end{array}$ & $\begin{array}{l}\text { stable for at } \\
\text { least } 1 \text { week }\end{array}$ \\
\hline
\end{tabular}

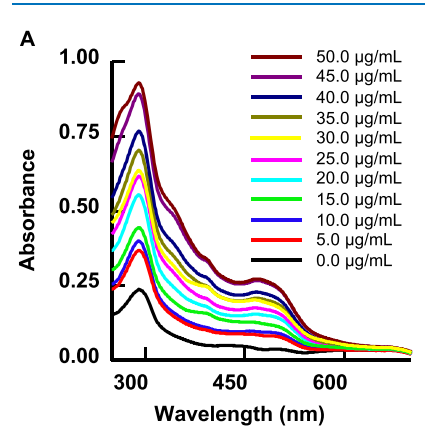

E

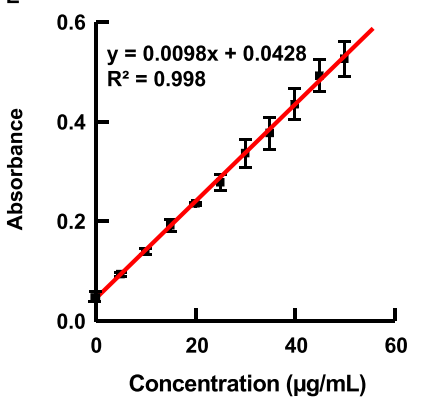

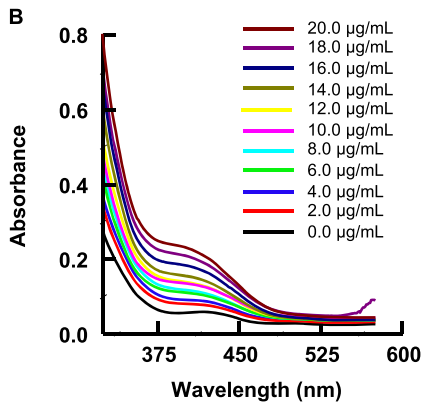

$\mathbf{F}$

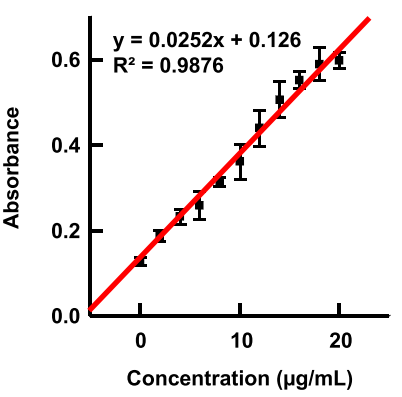

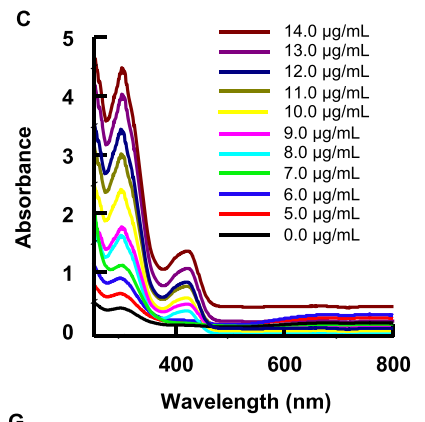

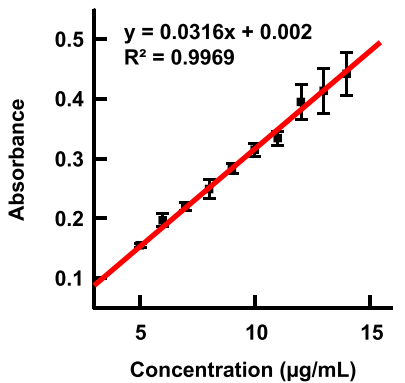

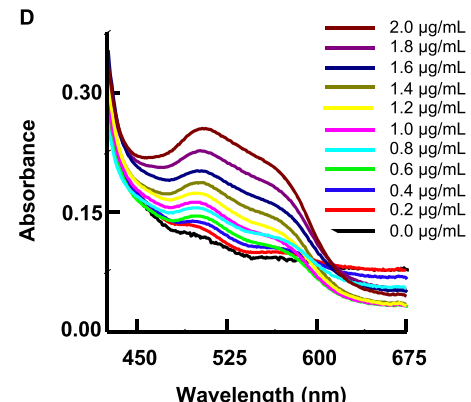

H

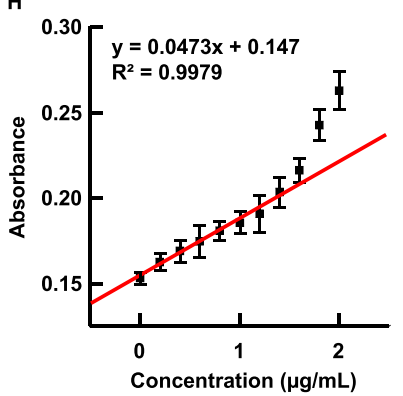

Figure 2. Representative absorbance spectrum of cortisol in various reagents and their concentration curves: $(A, E)$ sulfuric acid reagent with characteristic peaks at 290,394, and $470 \mathrm{~nm}$. The concentration curve plotted at $394 \mathrm{~nm}$. (B,F) AuNP Porter-Silber reagent with the characteristic peak at $410 \mathrm{~nm}$. (C,G) Prussian blue reagent with characteristic peaks at 300, 410, and $669 \mathrm{~nm}$. The concentration curve plotted at $669 \mathrm{~nm}$. (D,H) Blue tetrazolium reagent with the characteristic peak at $510 \mathrm{~nm}$.

color changes that are capable of visual inspection of cortisol concentration in the system (Figures 1 and S2). In Table 1, the characteristic absorption peak, limit of detection (LoD), time to color development, color change, and color stability are summarized for each method tested. Two types of LoDs are given: analytical and experimental, both of which are described in our Materials and Methods section. Characteristic absorption peaks from Prussian blue and blue tetrazolium methods differed slightly from previous reports. Singh and Verma reported a single absorbance peak at $780 \mathrm{~nm}$ using Prussian blue, while this study found two peaks, one at $300 \mathrm{~nm}$ and the other at $670 \mathrm{~nm} .^{21}$ Multiple studies report a peak at $525 \mathrm{~nm}$ for blue tetrazolium; however, our results show a peak at $510 \mathrm{~nm} .{ }^{19,20}$ The reason for both discrepancies is the difference in the solvent system. ${ }^{39}$ The blue tetrazolium method has the lowest LoD at $97 \mathrm{ng} / \mathrm{mL}$. The AuNP PorterSilber and blue tetrazolium methods are the only two capable of detecting cortisol within normal human sweat levels (8-142 $\mathrm{ng} / \mathrm{mL}){ }^{4}$ The time to color development listed in Table 1 is an approximation of the time it would take for the reagent to form an absorption peak discernible by the spectrometer. The time to color development appears to be dependent on the amount of cortisol added to the solution. Higher concentrations of cortisol produce more chromogens within a given time and therefore decrease color development time. Color stability also varies greatly among the methods. Cortisol in sulfuric acid causes a rapid and progressive change to the absorption magnitude. In agreement with previous reports, the Porter-Silber reagent itself becomes cloudy and opaque after about $24 \mathrm{~h}$, which interferes with spectroscopic analysis. ${ }^{40}$ The cloudiness is most likely due to the deterioration of phenylhydrazine, as it is both light and air sensitive. Prussian blue was stable for a week with no change in absorption and may last much longer. Baseline solution of the blue tetrazolium reagent also turns magenta, where after $12 \mathrm{~h}$, solutions with and without cortisol are nearly indistinguishable.

The characteristic peaks and the resulting concentration curve of various colorimetric analyses are shown in Figure 2 for further quantitative spectroscopic determination. For sulfuric acid-cortisol experiments, the color change is immediate upon adding cortisol to sulfuric acid and continues to increase absorbance indefinitely. The full absorbance spectrum of this reaction at 5 min post mixing presents peaks at 290, 394, and $480 \mathrm{~nm}$ while with increased intensity with respect to increased 

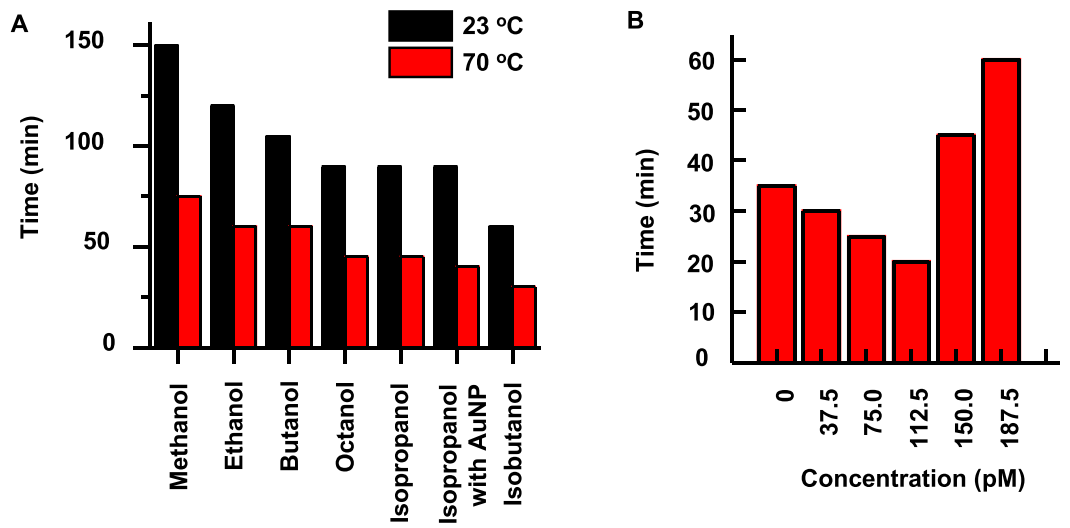

Figure 3. Effect of carbon content of alcohol and AuNPs on the color development time of Porter-Silber reaction: (A) time to initial color development at 23 and $70{ }^{\circ} \mathrm{C}$ with respect to increasing carbon content of the alcohol component of the Porter-Silber reaction. (B) Increase of AuNPs causes a decrease in color development time of the Porter-Silber reagent until the AuNP concentration exceeded 112.5 pM. The absorbance was determined at $410 \mathrm{~nm}$ in 5 min intervals as a function of AuNP concentration. The time at which each reaction reached an absorbance of 0.70 was recorded.

cortisol concentration (Figure 2A). A calibration curve was obtained at $394 \mathrm{~nm}$, and the LoD was calculated to be 3.00 $\mu \mathrm{g} / \mathrm{mL}$ with a sensitivity of $0.0098 \mathrm{Abs}(\mu \mathrm{g} / \mathrm{mL})^{-1}$ (Figure $2 \mathrm{E})$. Color stability of the sulfuric acid method is highly unstable and never reaches a plateau even after $100 \mathrm{~min}$ (Figure S3A). Overall, the sulfuric acid method is the simplest and quickest test. However, because of its LoD being in the upper range of human levels and spontaneous gradual color changes, this method functions better as a qualitative test rather than a quantitative test.

The traditional Porter-Silber reagent requires a minimum of $2 \mathrm{~h}$ to complete the reaction at room temperature. The previously reported strategy to increase the reaction rate was to increase the temperature at which the reaction took place to 70 ${ }^{\circ} \mathrm{C}$. ${ }^{15}$ However, for potential wearable biosensor applications, simply increasing the temperature to $70{ }^{\circ} \mathrm{C}$ would be inapplicable. Investigations into the mechanism of the Porter-Silber reaction replaced ethanol with methanol to slow down the reaction, which shows that the carbon content of the alcohol would have a large effect on the reaction rate. ${ }^{41}$ Figure $3 \mathrm{~A}$ and Table S1 show a comparison of different alcohols as a solvent system and their effect on the reaction rate. Lower carbon content alcohols, such as methanol and ethanol, require a longer time to complete the reaction as opposed to higher carbon content alcohols. Octanol exhibits a $33 \%$ decrease in reaction time, while butanol exhibits a $17 \%$ decrease. Isomers of alcohols also increase the reaction rate. Both isopropanol and isobutanol outperform their primary alcohol counterparts, nearly halving the total time to full color development where absorbance maxima began to plateau. Isopropanol yields optimum results for bioanalysis because of the solubility of isopropanol in dilute sulfuric acid, which also requires for Porter-Silber reaction. Octanol and isobutanol are immiscible with dilute sulfuric acid and produce a distinct phase separation (Figure S4).

Introduction of AuNPs improves the reaction rate of the Porter-Silber reaction assisted by catalytic reaction. ${ }^{42}$ However, an excess amount of AuNPs (concentrations > $112.5 \mathrm{pM}$ ) decreases the reaction time further without inhibiting the LoD or distorting the characteristic peak. With optimal AuNPs, the time to initial color development has been reduced nearly by half, from 120 to $60 \mathrm{~min}$ at room temperature. At $70{ }^{\circ} \mathrm{C}$, the reduction is from 60 to $30 \mathrm{~min}$.
The AuNP solution itself exhibits a red-salmon color, which has an absorption peak at $560 \mathrm{~nm}$. However, low concentration of the AuNP $(<100 \mathrm{pM})$ exhibited no interference in the final color development and the AuNP characteristic peak was nearly undetectable. Simply increasing the concentration of AuNPs does not necessarily mean faster color development. In concentrations larger than $150 \mathrm{pM}$, the AuNPs hinder the reaction, which results in a lengthier color development time than the traditional Porter-Silber solution (Figure 3B).

Figure 2B,F shows the results of the AuNP Porter-Silber reagent experiments that use isopropanol as a solvent, which is an optimized, improved, and modified Porter-Silber analysis. The reagent turns from clear to a translucent yellow and exhibits a maximum absorbance peak at $410 \mathrm{~nm}$, which increases in amplitude with respect to increased concentrations of cortisol. Plotting the absorbance peak against cortisol concentration at $30 \mathrm{~min}$ yields the calibration curve of $y=$ $0.0252 x+0.126\left(R^{2}=0.988\right)$. The $410 \mathrm{~nm}$ peak reaches a stable maximum and maintained color presentation after approximately $2 \mathrm{~h}$.

As a nontoxic dye commonly used in paints, Prussian blue poses a promising alternative, whereas previous methods require highly acidic and cytotoxic solvents. The solution begins at a pale yellow and changes to a green-blue. The absorption spectrum of the solution yields two peaks at 300 and $669 \mathrm{~nm}$. The absorption maxima of both peaks increase with respect to increasing concentrations of cortisol, as shown in Figure 2C,G. Although the relationship between the absorption value and cortisol concentration is linear, the standard deviation of the curve is quite high. The unreliability of this method arises because of the difference in particle size of the resulting colloidal solution. The oxidative state of iron $\left(\mathrm{Fe}^{2+}\right.$ vs $\left.\mathrm{Fe}^{3+}\right)$ drastically changes the particle size of the colloid, about 40-100 $\mathrm{nm}$ in diameter, and it therefore changes the absorption peaks. ${ }^{34}$ Nevertheless, the Prussian blue method is still capable of determining cortisol in various concentrations $(1.0-15 \mu \mathrm{g} / \mathrm{mL})$.

Blue tetrazolium in conjunction with tetramethylammonium hydroxide yields a magenta solution. In this study, we used methanol as a primary solvent, while previous studies used either ethanol or dichloromethane. ${ }^{19,20}$ The use of methanol decreased the reaction time (approximately 25\% faster than ethanol) with no changes in sensitivity. Figure $2 \mathrm{D}, \mathrm{H}$ shows 

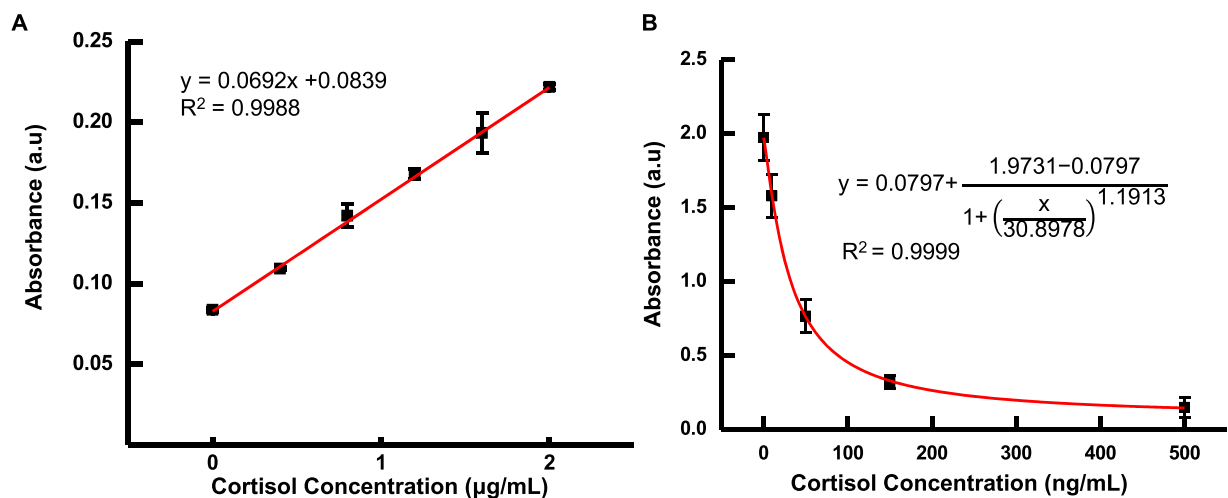

Figure 4. Analytical performance comparison of the (A) blue tetrazolium reagent and (B) ELISA for monitoring cortisol in a different range of concentrations: (A) blue tetrazolium capable of detecting $\mu \mathrm{g} / \mathrm{mL}$ of ranges and an experimental LoD of $0.045 \mu \mathrm{g} / \mathrm{mL}$. (B) ELISA method offers more sensitivity than the blue tetrazolium method. However, its linear dynamic range is significantly lower, which may fit for $\mathrm{ng} / \mathrm{mL}$ determination.

that the peak absorption increases with respect to increasing concentrations of cortisol. The calibration curve shows linear relationship with a sensitivity of $0.038 \mathrm{Abs}(\mu \mathrm{g} / \mathrm{mL})^{-1}$ and dynamic range from 0.2 to $1.6 \mu \mathrm{g} / \mathrm{mL}$. Both the blank and cortisol solution turn magenta because the reaction depends on the hydrolysis rate of the cyclic diacetyl in blue tetrazolium. ${ }^{43}$ Although absorbance saturates over time, the time until the full color development varies significantly as a function of cortisol concentration. To stabilize the reaction and to slow the redox process, the reaction can be carried out in a solvent with a large dielectric constant, such as water. The largest advantage of the blue tetrazolium method is its quick color development, which is an advancement for an industry pushing for rapid result diagnostics.

All four methods of determining cortisol in the complex biofluid matrix, such as saliva and sweat, resulted in a similar analytical response. Quantitative analytical analysis in sensitivity, LoD, and dynamic ranges demonstrate insignificant changes with artificial sweat and saliva (Figure S5). Overall, blue tetrazolium is the best overall method of detecting cortisol because of rapid reaction rate, low toxicity, low LoD within physiologically relevant levels, and a single peak for simple analysis, which are all beneficial for wearable optoelectronic applications and stress monitoring.

Blue tetrazolium methods may be comparable to the goldstandard ELISA technique for certain biosensor applications. It is noteworthy that the blue tetrazolium method's greatest asset is the simplicity of its one-step reaction, which completes in under $10 \mathrm{~min}$; it is far easier and faster than ELISA methods. Additionally, blue tetrazolium has a significantly greater range of detection, which is useful for diagnosing those with disorders such as Cushing's syndrome, which produces characteristically high levels of cortisol in patients. In Figure 4, both blue tetrazolium and ELISA concentration curves made using artificial sweat are shown. For blue tetrazolium in artificial sweat, the LoD increases slightly from 97 to $146 \mathrm{ng} /$ $\mathrm{mL}$, which is expected to be because of the sweat's composition comprising mostly water. ELISA is still the gold standard in laboratory settings; however, we believe it is not as appealing as blue tetrazolium in point-of-care applications.

To demonstrate the viability of blue tetrazolium as a diagnostic tool, we analyzed apocrine and eccrine sweat samples from different subjects $(n=21)$ and correlated their sweat cortisol concentration to their psychological stress levels. Subjects self-reported their psychological distress using the
Kessler Psychological Distress Scale (K10) with 40 min of exercising. K10 scores range from 10 to 50, with scores under 20, 20-24, 25-29, and over 30 correlating with low, mild, moderate, and severe anxiety and stress, respectively. ${ }^{44}$ Overall, both men and women qualitatively exhibited a positive correlation between their K10 score and absorbance (cortisol concentration). The resulting sweat cortisol concentration is higher than normal cortisol concentration, which is attributed to the physical stress subjects had to undergo to produce sweat. Normal pituitary response to psychological or physical stress is to release cortisol. Therefore, the greater the stress in aptitude, the higher the basal cortisol concentration in the body. The results show a significant difference in cortisol concentration in apocrine sweat produced by men versus women. As shown in Figure 4, male participants $(n=6)$ had an average apocrine cortisol absorbance of $0.136 \pm 0.028$, whereas female participants $(n=15)$ had an average of $0.106 \pm 0.031$. Statistical analysis uses a two-tailed $t$-test, which reveals a $p$ value of 0.042 that indicates a significant difference between the two groups in the $95 \%$ confidence interval. Likewise, there is a highly significant difference between apocrine and eccrine cortisol concentrations. Apocrine sweat samples have an average of $0.12 \pm 0.032$, while eccrine samples have an average of $0.072 \pm 0.012$ (Figure 5). A two-tailed paired $t$-test results in a $p$-value of 0.000042 . Based on our calibration curves, the basal cortisol concentration varies greatly between

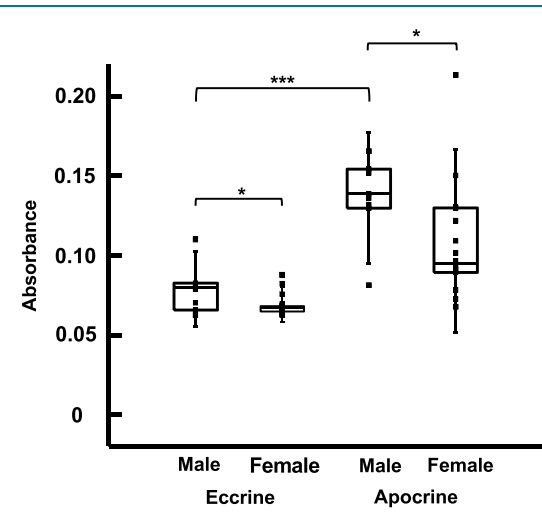

Figure 5. Apocrine and eccrine cortisol concentration of male and female participants: boxplot analysis shows a significant difference ( $p$ value $=0.042$ ) between male and female cortisol levels and a highly significant difference ( $p$-value $=0.0000042)$ between apocrine and eccrine sweat in both males and females. 
subjects, with a range of $13.3-443 \mu \mathrm{g} / \mathrm{mL}$. Further studies are needed with more subjects to confirm our results and to compare them to existing methods, such as ELISA, or more sophisticated instrumental analysis, such as high-performance liquid chromatography-mass spectrometry.

The differences between men and women and apocrine and eccrine could be explained by the pituitary gland's regulation of cortisol. The main regulatory organ of cortisol is the pituitary gland, which diverges in development when males and females go through puberty. ${ }^{45}$ The pituitary gland also has a role in regulating sweat. Adenylate cyclase, a second messenger released by the pituitary gland, promotes different levels of eccrine sweat secretion in men versus women. ${ }^{46}$ It is also possible that cortisone and corticosterone, both metabolites of cortisol, occur in higher concentrations in men. However, any interference due to each steroid would be minimal because of its inherently lower concentration in the body and delayed because of the metabolizing process. ${ }^{47}$ The exact cause of the difference between groups needs to be further investigated. Overall, this pilot study shows the great potential that such methods have for monitoring and diagnosing cortisol-related illnesses.

\section{CONCLUSIONS}

All the methods presented in this study can be used as a fast and simple qualitative and quantitative diagnostic tool for the detection of cortisol concentration in biofluids such as saliva and sweat. For qualitative assessment, the pure sulfuric reaction can be used like a $\mathrm{pH}$ litmus test and provide a good approximation of cortisol concentration in a short amount of time. For quantitative measurements, the AuNP Porter-Silber or the blue tetrazolium reaction with methanol grants in situ analysis with less concern of cytotoxicity. Such cortisol colorimetric methods have the benefit over electrochemical sensors by not relying on electronic components and can be easily analyzed by the naked eye, which provides intuitive understanding of the cortisol level. These colorimetric analyses are especially beneficial in analytical settings with limited access to state-of-the-art instrumentations. Tests performed on common noninvasive biofluids, such as sweat and saliva, disclosed that no excipients or other biomarkers interfere with these reactions. The pilot study revealed a previously unreported difference in cortisol levels between apocrine and eccrine sweat glands and between men and women ( $p$-value $=0.000042$ and 0.042 , respectively). Although other steroid hormones, such as testosterone and estrogen, do not affect the reaction, it is unknown whether or not more closely related steroids, such as cortisone or corticosterone, may influence spectrophotometric results. Therefore, research into the selectivity of each method will be further studied. In our future work, we will incorporate colorimetric methods into a single, skin-interfaced microfluidics biosensor to provide both qualitative and quantitative measurements to monitor stress and stress-related illnesses. ${ }^{31,48}$ The development of a smartphone application that enables the user's phone camera in place of a spectrometer and reads pixel values in lieu of absorbance is underway. ${ }^{49,50}$ The simplicity of such low-cost methods facilitates the widespread use of such devices of disposable, low-cost wearable devices.

\section{MATERIALS AND METHODS}

For a detailed list of the materials and their manufacturer, please refer to the Supporting Information section of this paper.

Preparation of Cortisol Stock. The cortisol stock solutions were prepared fresh every week. For analytical assessments in general, the solution contained $20 \pm 0.1 \mathrm{mg}$ of hydrocortisone dissolved in $10 \mathrm{~mL}$ of absolute ethanol (5.5 $\mathrm{mM}$ ). This solution was primarily used to obtain calibration curves and absorbance spectra of the different methods. To determine cortisol contents in artificial biofluids, the calibration curves were constructed with solutions that contain $4.0 \pm 0.1 \mathrm{mg}(0.55 \mathrm{mM})$ and $2.0 \pm 0.1 \mathrm{mg}(0.28 \mathrm{mM})$ of hydrocortisone in $20 \mathrm{~mL}$ of artificial saliva and artificial sweat, respectively.

Acid-Induced Fluorescence Method. Appropriate amounts of cortisol stock were added directly to concentrated sulfuric acid into quartz cuvettes. The solution was then well mixed with pipettes. Measurements were taken $5 \mathrm{~min}$ after mixing.

Porter-Silber Reagent Method with AuNPs. The traditional Porter-Silber reagent was prepared according to the original article's specifications. ${ }^{14}$ Furthermore, we studied various solvent systems while replacing ethanol in the original reagent to improve analytical performances. These alcohol solvents include methanol, isopropanol, butanol, isobutanol, and octanol. These Porter-Silber reagents, which contain 4 $\mathrm{mL}$ of concentrated sulfuric acid, $2.5 \mathrm{~mL}$ of distilled water, 3.5 $\mathrm{mL}$ of alcohol, and $20 \mathrm{mg}$ of phenylhydrazine, were prepared fresh daily. Additional tests were carried out with solutions that contain AuNPs. In this solution, $2.4 \mathrm{~mL}$ of distilled water with $100 \mu \mathrm{L}$ of AuNPs was used instead of $2.5 \mathrm{~mL}$ of DI water. To create a calibration curve, appropriate amounts of cortisol stock were added to the Porter-Silber reagents at room temperature $\left(23{ }^{\circ} \mathrm{C}\right)$ for approximately $1 \mathrm{~h}$ until color formation. Measurements were taken every $15 \mathrm{~min}$ after the addition of cortisol.

Prussian Blue Methods. A modified method was used to form a light-toned Prussian blue $\left(\mathrm{C}_{18} \mathrm{Fe}_{7} \mathrm{~N}_{18}\right) .{ }^{38} \mathrm{An}$ iron(III) solution was prepared by adding $10 \mathrm{mg}$ of iron(III) chloride to $20 \mathrm{~mL}$ of ethanol. Potassium hexacyanoferrate(III) solutions were similarly prepared by adding $13.5 \mathrm{mg}$ of potassium hexacyanoferrate(III) to $20 \mathrm{~mL}$ of ethanol. Cortisol stock was first mixed with equal parts of sulfuric acid and iron(III) solution $(2 \mathrm{~mL}$ each $)$ to oxidize the corticosteroid. After $5 \mathrm{~min}$ of mixing, $0.5 \mathrm{~mL}$ of potassium hexacyanoferrate solution was added. The solution was then put in a water bath at $70{ }^{\circ} \mathrm{C}$ until color formation. Measurements were taken every $10 \mathrm{~min}$ after the addition of cortisol.

Blue Tetrazolium Methods. A 1\% v/v tetramethylammonium hydroxide solution $\left(\mathrm{N}\left(\mathrm{CH}_{3}\right)^{4+} \mathrm{OH}^{-}\right)$was prepared by diluting $5 \mathrm{~mL}$ of the aqueous solution in $45 \mathrm{~mL}$ of methanol. A second solution that contains $100 \mathrm{mg}$ of blue tetrazolium dissolved in $50 \mathrm{~mL}$ of methanol was made. Equal parts of both solutions were then mixed along with appropriate amounts of cortisol stock at room temperature. Measurements were taken every $10 \mathrm{~min}$ after the addition of cortisol.

Spectroscopy Analysis. For each colorimetric method, a concentration curve was constructed by adding appropriate amounts of cortisol stock to the respective reagent. Measurements were taken at predetermined intervals in $1 \mathrm{~cm}^{2}$ quartz cuvettes (VWR) and using a UV-Vis Spectrometer (Cary 60, 
Agilent Technologies) from 300 to $800 \mathrm{~nm}$. All solutions were measured against a baseline solution, which includes all components of the respective reagent without the addition of cortisol.

Small-n Human Pilot Study. The research protocol was approved by Binghamton University's Institutional Review Board (MOD00000592). Participants were healthy subjects aged between 19 and 30, of which seven are males and 14 are females. Sweat samples collected from 21 subjects were analyzed using the blue tetrazolium method. Measurements were taken at $10 \mathrm{~min}$ at $510 \mathrm{~nm}$ wavelength. Subjects completed the Kessler Psychological Distress Scale (K10) prior to exercise. Cortisol concentrations from both armpits and lower back were then compared to the scores obtained through the K10 test. The full sweat study design is outlined in the Supporting Information, Figure S1.

\section{ASSOCIATED CONTENT}

\section{SI Supporting Information}

The Supporting Information is available free of charge at https://pubs.acs.org/doi/10.1021/acsomega.0c00498.

Schematic illustration of human sweat pilot study; colorimetric reactions of different reagents with cortisol; time to full color development of various reagents; Porter-Silber reaction with various alcohol solvents; Porter-Silber reagent with octanol; comparison of concentration curves derived from cortisol stock, artificial saliva, and artificial sweat; representative absorbance spectrum of human sweat in blue tetrazolium reagent $(\mathrm{PDF})$

\section{AUTHOR INFORMATION}

\section{Corresponding Author}

Ahyeon Koh - Department of Biomedical Engineering, Binghamton University - State University of New York, Binghamton, New York 13902, United States; (1) orcid.org/ 0000-0003-2721-2321; Phone: +1 (607) 777-5422; Email: akoh@binghamton.edu; Fax: +1 (607) 777-5780

\section{Authors}

Ethan Tu - Department of Biomedical Engineering, Binghamton University-State University of New York, Binghamton, New York 13902, United States; Department of Biomedical Engineering, Michigan State University, East Lansing, Michigan 48824, United States

Paul Pearlmutter - Department of Biomedical Engineering, Binghamton University-State University of New York, Binghamton, New York 13902, United States

Michelle Tiangco - Department of Biomedical Engineering, Binghamton University - State University of New York, Binghamton, New York 13902, United States

Gia Derose - Health and Wellness Studies Department, Binghamton University-State University of New York, Binghamton, New York 13902, United States

Lina Begdache - Health and Wellness Studies Department, Binghamton University - State University of New York, Binghamton, New York 13902, United States

Complete contact information is available at: https://pubs.acs.org/10.1021/acsomega.0c00498

\section{Notes}

The views and conclusions contained herein are those of the authors and should not be interpreted as necessarily representing the official policies or endorsements, either expressed or implied, of Air Force Research Laboratory, the U.S. Government, or SEMI-FlexTech.

The authors declare no competing financial interest.

\section{ACKNOWLEDGMENTS}

This research is supported by the Small Scale Systems Integration and Packaging (S3IP) Center of Excellence and SUNY Binghamton University Start-Up funds. This material is based on research sponsored by Air Force Research Laboratory under the agreement number FA8650-18-2-5402. The U.S. government is authorized to reproduce and distribute reprints for government purposes notwithstanding any copyright notation thereon.

\section{REFERENCES}

(1) de Kloet, E. R.; Joëls, M.; Holsboer, F. Stress and the Brain: From Adaptation to Disease. Nat. Rev. Neurosci. 2005, 6, 463-475.

(2) Dedovic, K.; Duchesne, A.; Andrews, J.; Engert, V.; Pruessner, J. C. The Brain and the Stress Axis: The Neural Correlates of Cortisol Regulation in Response to Stress. NeuroImage 2009, 47, 864-871.

(3) Kaushik, A.; Vasudev, A.; Arya, S. K.; Pasha, S. K.; Bhansali, S. Recent Advances in Cortisol Sensing Technologies for Point-of-Care Application. Biosens. Bioelectron. 2014, 53, 499-512.

(4) Russell, E.; Koren, G.; Rieder, M.; Van Uum, S. H. M. The Detection of Cortisol in Human Sweat: Implications for Measurement of Cortisol in Hair. Ther. Drug Monit. 2014, 36, 30-34.

(5) Phillips, D. I. W.; Barker, D. J. P.; Fall, C. H. D.; Seckl, J. R.; Whorwood, C. B.; Wood, P. J.; Walker, B. R. Elevated Plasma Cortisol Concentrations: A Link between Low Birth Weight and the Insulin Resistance Syndrome? J. Clin. Endocrinol. Metab. 1998, 83, 757-760.

(6) Vining, R. F.; McGinley, R. A.; Maksvytis, J. J.; Ho, K. Y. Salivary Cortisol: A Better Measure of Adrenal Cortical Function than Serum Cortisol. Ann. Clin. Biochem. 1983, 20, 329-335.

(7) McEwen, B. S. Cortisol, Cushing's Syndrome, and a Shrinking Brain-New Evidence for Reversibility. J. Clin. Endocrinol. Metab. 2002, 87, 1947-1948.

(8) Edwards, O. M.; Courtenay-Evans, R. J.; Galley, J. M.; Hunter, J.; Tait, A. D. Changes in Cortisol Metabolism Following Rifampicin Therapy. Lancet 1974, 2, 548-551.

(9) Goldzieher, J. W.; Bodenchuk, J. M.; Nolan, P. Fluorescence Reactions of Steroids. Anal. Chem. 1954, 26, 853-856.

(10) Sweat, M. L. Sulfuric Acid-Induced Fluorescence of Corticosteroids. Anal. Chem. 1954, 26, 773-776.

(11) Kalant, H. Chromogenic and Fluorogenic Reactions of Adrenocortical and Other Steroids in Concentrated Acids. Biochem. J. 1958, 69, 79-93.

(12) Zimmermann, W. Eine Farbreaktion Der Sexualhormone Und Ihre Anwendung Zur Quantitativen Colorimetrischen Bestimmung. Hoppe Seylers Z. hysiol.Chem. 1935, 233, 257-264.

(13) James, V. H. T.; De Jong, M. The Use of Tetramethylammonium Hydroxide in the Zimmermann Reaction. J. Clin. Pathol. 1961, 14, 425-430.

(14) Porter, C. C.; Silber, R. H. A Quantitative Color Reaction for Cortisone and Related 17,21-Dihydroxy-20-Ketosteroids. J. Biol. Chem. 1950, 185, 201-207.

(15) Silber, R. H.; Porter, C. C. Determination of 17,21-Dihydroxy20-Ketosteroids in Urine and Plasma. Methods of Biochemical Analysis; John Wiley \& Sons, Ltd, 2006; Vol. 4, pp 139-169.

(16) Simon, M.; Babich-Armstrong, M. 17-Hydroxycorticosteroids in Urine. Clin. Chim. Acta 1980, 103, 101-108.

(17) Furuya, E.; Graef, V.; Nishikaze, O. An Improved Method Based on the Porter-Silber Reaction for Determining 17-Hydroxycorticosteroids in Urine. Anal. Biochem. 1978, 90, 644-650. 
(18) Clark, I. Colorimetric Reaction for the Estimation of Cortisone, Hydrocortisone, Aldosterone and Related Steroids. Nature 1955, 175, 123-124.

(19) Guttman, D. E. Analysis of Steroids in Mixtures Using the Kinetics of Blue Tetrazolium Reduction. J. Pharm. Sci. 1966, 55, 919922.

(20) Graham, R. E.; Biehl, E. R.; Kenner, C. T. Rapid Blue Tetrazolium Procedure for Analysis of Corticosteroids in Pharmaceutical Preparations. J. Pharm. Sci. 1978, 67, 792-795.

(21) Singh, D. K.; Verma, R. Spectrophotometric Determination of Corticosteroids and Its Application in Pharmaceutical Formulation. Iran. J. Pharmacol. Ther.. 2008, 7 (), 61-65. http://ijpt.iums.ac.ir/ article-1-155-en.html.

(22) Singh, A.; Kaushik, A.; Kumar, R.; Nair, M.; Bhansali, S. Electrochemical Sensing of Cortisol: A Recent Update. Appl. Biochem. Biotechnol. 2014, 174, 1115-1126.

(23) Gao, Z.; Deng, K.; Wang, X.-D.; Miró, M.; Tang, D. HighResolution Colorimetric Assay for Rapid Visual Readout of Phosphatase Activity Based on Gold/Silver Core/Shell Nanorod. ACS Appl. Mater. Interfaces 2014, 6, 18243-18250.

(24) Gao, Z.; Tang, D.; Tang, D.; Niessner, R.; Knopp, D. TargetInduced Nanocatalyst Deactivation Facilitated by Core@Shell Nanostructures for Signal-Amplified Headspace-Colorimetric Assay of Dissolved Hydrogen Sulfide. Anal. Chem. 2015, 87, 10153-10160.

(25) Gao, Z.; Xu, M.; Hou, L.; Chen, G.; Tang, D. Magnetic BeadBased Reverse Colorimetric Immunoassay Strategy for Sensing Biomolecules. Anal. Chem. 2013, 85, 6945-6952.

(26) Ren, R.; Cai, G.; Yu, Z.; Zeng, Y.; Tang, D. MetalPolydopamine Framework: An Innovative Signal-Generation Tag for Colorimetric Immunoassay. Anal. Chem. 2018, 90, 11099-11105.

(27) Pinto, V.; Sousa, P.; Catarino, S. O.; Correia-Neves, M.; Minas, G. Microfluidic Immunosensor for Rapid and Highly-Sensitive Salivary Cortisol Quantification. Biosens. Bioelectron. 2017, 90, 308313.

(28) Munje, R. D.; Muthukumar, S.; Panneer Selvam, A.; Prasad, S. Flexible Nanoporous Tunable Electrical Double Layer Biosensors for Sweat Diagnostics. Sci. Rep. 2015, 5, 14586.

(29) Schazmann, B.; Morris, D.; Slater, C.; Beirne, S.; Fay, C.; Reuveny, R.; Moyna, N.; Diamond, D. A Wearable Electrochemical Sensor for the Real-Time Measurement of Sweat Sodium Concentration. Anal. Methods 2010, 2, 342-348.

(30) Gao, W.; Emaminejad, S.; Nyein, H. Y. Y.; Challa, S.; Chen, K.; Peck, A.; Fahad, H. M.; Ota, H.; Shiraki, H.; Kiriya, D.; et al. Fully Integrated Wearable Sensor Arrays for Multiplexed in Situ Perspiration Analysis. Nature 2016, 529, 509-514.

(31) Choi, J.; Bandodkar, A. J.; Reeder, J. T.; Ray, T. R.; Turnquist, A.; Kim, S. B.; Nyberg, N.; Hourlier-Fargette, A.; Model, J. B.; Aranyosi, A. J.; et al. Soft, Skin-Integrated Multifunctional Microfluidic Systems for Accurate Colorimetric Analysis of Sweat Biomarkers and Temperature. ACS Sens. 2019, 4, 379-388.

(32) Zhao, J.; Guo, H.; Li, J.; Bandodkar, A. J.; Rogers, J. A. BodyInterfaced Chemical Sensors for Noninvasive Monitoring and Analysis of Biofluids. Trends Chem. 2019, 1, 559-571.

(33) Kim, K. S.; Lim, S. R.; Kim, S.-E.; Lee, J. Y.; Chung, C.-H.; Choe, W.-S.; Yoo, P. J. Highly Sensitive and Selective Electrochemical Cortisol Sensor Using Bifunctional Protein Interlayer-Modified Graphene Electrodes. Sens. Actuators, B 2017, 242, 1121-1128.

(34) Parlak, O.; Keene, S. T.; Marais, A.; Curto, V. F.; Salleo, A. Molecularly Selective Nanoporous Membrane-Based Wearable Organic Electrochemical Device for Noninvasive Cortisol Sensing. Sci. Adv. 2018, 4, No. eaar2904.

(35) Kim, S. B.; Koo, J.; Yoon, J.; Hourlier-Fargette, A.; Lee, B.; Chen, S.; Jo, S.; Choi, J.; Oh, Y. S.; Lee, G.; et al. Soft, Skin-Interfaced Microfluidic Systems with Integrated Enzymatic Assays for Measuring the Concentration of Ammonia and Ethanol in Sweat. Lab Chip 2020, 20, 84-92.

(36) Zhang, Y.; Guo, H.; Kim, S. B.; Wu, Y.; Ostojich, D.; Park, S. H.; Wang, X.; Weng, Z.; Li, R.; Bandodkar, A. J.; et al. Passive Sweat Collection and Colorimetric Analysis of Biomarkers Relevant to
Kidney Disorders Using a Soft Microfluidic System. Lab Chip 2019, 19, 1545-1555.

(37) Ghaffari, R.; Choi, J.; Raj, M. S.; Chen, S.; Lee, S. P.; Reeder, J. T.; Aranyosi, A. J.; Leech, A.; Li, W.; Schon, S.; et al. Soft Wearable Systems for Colorimetric and Electrochemical Analysis of Biofluids. Adv. Funct. Mater. 2019, 1907269.

(38) Ozeki, T.; Matsumoto, K.; Hikime, S. Photoacoustic Spectra of Prussian Blue and Photochemical Reaction of Ferric Ferricyanide. Anal. Chem. 1984, 56, 2819-2822.

(39) Graham, R. E.; Biehl, E. R.; Kenner, C. T. Effect of Solvent on Tetrazolium Reaction. J. Pharm. Sci. 1976, 65, 1048-1053.

(40) Pinkus, G. S.; Pinkus, J. L. Isolation of Urinary 17-Ketosteroid Conjugates by Ammonium Sulfate Precipitation. Clin. Chem. 1971, 17, 37-41.

(41) Lewbart, M. L.; Mattox, V. R. The Mechanism of the PorterSilber Reaction. I. Rearrangement of the Dihydroxyacetone Group of Steroids1. J. Org. Chem. 1964, 29, 513-521.

(42) Ide, M. S.; Davis, R. J. The Important Role of Hydroxyl on Oxidation Catalysis by Gold Nanoparticles. Acc. Chem. Res. 2014, 47, 825-833.

(43) Oteiza, R. M.; Wooten, R. S.; Kenner, C. T.; Graham, R. E.; Biehl, E. R. Kinetics and Mechanism of Blue Tétrazolium Reaction with Corticosteroids. J. Pharm. Sci. 1977, 66, 1385-1388.

(44) Krynen, A. M.; Osborne, D.; Duck, I. M.; Houkamau, C. A.; Sibley, C. G. Measuring Psychological Distress in New Zealand: Item Response Properties and Demographic Differences in the Kessler-6 Screening Measure. N. Z. J. Psychol. 2013, 42, 15.

(45) MacMaster, F. P.; Keshavan, M.; Mirza, Y.; Carrey, N.; Upadhyaya, A. R.; El-Sheikh, R.; Buhagiar, C. J.; Taormina, S. P.; Boyd, C.; Lynch, M.; et al. Development and Sexual Dimorphism of the Pituitary Gland. Life Sci. 2007, 80, 940-944.

(46) Sasaki, S.; Watanabe, J.; Ohtaki, H.; Matsumoto, M.; Murai, N.; Nakamachi, T.; Hannibal, J.; Fahrenkrug, J.; Hashimoto, H.; Watanabe, H.; et al. Pituitary Adenylate Cyclase-Activating Polypeptide Promotes Eccrine Gland Sweat Secretion. Br. J. Dermatol. 2017, $176,413-422$.

(47) Morita, H.; Isomura, Y.; Mune, T.; Daido, H.; Takami, R.; Yamakita, N.; Ishizuka, T.; Takeda, N.; Yasuda, K.; Gomez-Sanchez, C. E. Plasma Cortisol and Cortisone Concentrations in Normal Subjects and Patients with Adrenocortical Disorders. Metab., Clin. Exp. 2004, 53, 89-94.

(48) Koh, A.; Kang, D.; Xue, Y.; Lee, S.; Pielak, R. M.; Kim, J.; Hwang, T.; Min, S.; Banks, A.; Bastien, P.; et al. A Soft, Wearable Microfluidic Device for the Capture, Storage, and Colorimetric Sensing of Sweat. Sci. Transl. Med. 2016, 8, 366ra165.

(49) Geng, Z.; Zhang, X.; Fan, Z.; Lv, X.; Su, Y.; Chen, H. Recent Progress in Optical Biosensors Based on Smartphone Platforms. Sensors 2017, 17, 2449.

(50) Rose, D. P.; Ratterman, M. E.; Griffin, D. K.; Hou, L.; KelleyLoughnane, N.; Naik, R. R.; Hagen, J. A.; Papautsky, I.; Heikenfeld, J. C. Adhesive RFID Sensor Patch for Monitoring of Sweat Electrolytes. IEEE Trans. Biomed. Eng. 2015, 62, 1457-1465. 